\title{
Overcoming innate immune barriers that impede AAV gene therapy vectors
}

\author{
Manish Muhuri, ${ }^{1,2,3}$ Yukiko Maeda, ${ }^{1,3,4}$ Hong Ma, ${ }^{1}$ Sanjay Ram, ${ }^{5}$ Katherine A. Fitzgerald, ${ }^{6}$ Phillip W.L. Tai, ${ }^{1,2,3}$ and Guangping Gao ${ }^{1,2,7}$ \\ ${ }^{1}$ Horae Gene Therapy Center, ${ }^{2}$ Department of Microbiology and Physiological Systems, ${ }^{3}$ VIDE Program, ${ }^{4}$ Department of Medicine, ${ }^{5}$ Division of Infectious Diseases and Immunology, ${ }^{6}$ Program in Innate \\ Immunity, Department of Medicine, and 'Li Weibo Institute for Rare Diseases Research, University of Massachusetts Medical School, Worcester, Massachusetts, USA
}

\begin{abstract}
The field of gene therapy has made considerable progress over the past several years. Adeno-associated virus (AAV) vectors have emerged as promising and attractive tools for in vivo gene therapy. Despite the recent clinical successes achieved with recombinant AAVs (rAAVs) for therapeutics, host immune responses against the vector and transgene product have been observed in numerous preclinical and clinical studies. These outcomes have hampered the advancement of AAV gene therapies, preventing them from becoming fully viable and safe medicines. The human immune system is multidimensional and complex. Both the innate and adaptive arms of the immune system seem to play a concerted role in the response against rAAVs. While most efforts have been focused on the role of adaptive immunity and developing ways to overcome it, the innate immune system has also been found to have a critical function. Innate immunity not only mediates the initial response to the vector, but also primes the adaptive immune system to launch a more deleterious attack against the foreign vector. This Review highlights what is known about innate immune responses against rAAVs and discusses potential strategies to circumvent these pathways.
\end{abstract}

\section{Introduction}

Adeno-associated viruses (AAVs) are small ( 26 nm), nonenveloped viruses that belong to the Parvoviridae family. AAVs are found naturally in multiple vertebrate species, including humans and nonhuman primates (1). Presently, 12 different AAV serotypes and more than 100 natural isolates have been identified (2). AAVs are nonpathogenic and possess relatively low immunogenicity. $\mathrm{AAV}$ is nonreplicating on its own, requiring other helper viruses, such as adenovirus and herpesvirus, to complete its life cycle. The 4.7-kb single-stranded genome, which encodes for four known open reading frames (rep, cap, assembly-activating protein [AAP], and the recently discovered membrane-associated accessory protein [MAAP]; refs. 3, 4), is flanked by two hairpin structures called inverted terminal repeats (ITRs). In the absence of helper viruses, AAV remains latent in the host cell as nonreplicating epichromosomal DNAs, or is integrated into the host cell genome (2). Multiple comprehensive reviews describe AAV's protein and genome structure $(3,5)$, the details of which will not be covered here.

Recombinant AAV (rAAV) vectors have emerged as one of the leading tools for facilitating gene therapeutics for rare monogenic diseases. A typical rAAV consists of a capsid that encapsidates a transgene expression cassette instead of the wild-type AAV pro-

Authorship note: $\mathrm{MM}$ and $\mathrm{YM}$ contributed equally to this work.

Conflict of interest: GG is a scientific cofounder of Voyager Therapeutics and Aspa Therapeutics, and holds equity in these companies. CG is an inventor on patents with potential royalties licensed to Voyager Therapeutics, Aspa Therapeutics, and other biopharmaceutical companies. PWLT is an inventor on patents with potential royalties licensed to Kanghong Therapeutics (patent application no. PCT/US2020/049243) KAF is an SAB member at Generation Bio and holds equity in this company.

Copyright: (c) 2021, American Society for Clinical Investigation.

Reference information: J Clin Invest. 2021;131(1):e143780.

https://doi.org/10.1172/JCl143780. tein coding sequences. The ITRs, which are essential for guiding genome replication and packaging during vector production, are the only sequences of viral origin residing in rAAVs. Owing to their ease of production and versatility in infecting both dividing and quiescent cells in diverse tissue types, rAAVs are extremely popular and considered relatively safe. The rAAV genome exists predominantly as an episome in the host cell nucleus, thereby establishing long-term transgene expression in nonreplicating cell types (6).

The host immune response is one of the most critical roadblocks limiting effective and long-term transgene expression (3, 5). Preexisting neutralizing antibodies (NAbs) against the AAV capsids are found in a large portion of the human population as a result of natural AAV infection $(7,8)$. NAbs can effectively bind and neutralize rAAV, blocking transduction in target cells (9). In addition, cellular immune responses against the rAAV capsid and/ or transgene can trigger a strong humoral immune response and elicit NAbs that prevent successful vector readministration (10). In addition, the capsid can trigger a cytotoxic T lymphocyte-mediated (CTL-mediated) response that leads to the loss of transgene expression (11). Furthermore, the transgene can also induce B cell- and T cell-mediated adaptive immune responses to generate transgene product-specific antibodies and CTLs. In the last few years, a series of studies have reported novel rAAV-related inflammatory toxicities in nonhuman primates (NHPs) and neonatal piglets (12). These include neuroinflammation in the spinal dorsal root ganglia after high-dose intrathecal administration, and acute thrombocytopenia and hepatic and renal toxicity after high-dose intravenous administration (13-16).

Most recently, three patients with X-linked myotubular myopathy $(\mathrm{XLMTM})$ who were given a high dose $\left(3 \times 10^{14}\right.$ genome copies $/ \mathrm{kg}$ ) of AT132 (rAAV8 expressing the therapeutic MTM1 transgene) experienced severe hepatobiliary disease, which culminated 
in their deaths (17-19). While the precise mechanisms that caused these toxicities are under investigation, one hypothesis attributes this effect to preexisting antibodies against AAV, resulting in activation of innate immunity and/or the classical pathway of the complement system. Two of the three patients who died experienced bacterial infections and sepsis, and all three reportedly had preexisting hepatobiliary disease that may have aggravated the challenge. In addition, these patients were at the higher end of the age cutoff and the lower range of normal body weight - factors that could further confound any proposed explanation (20). Several other adverse events of varying severity have occurred in patients receiving high systemic doses of rAAVs to treat spinal muscular atrophy type I, XLMTM, and Duchenne muscular dystrophy. These outcomes also seem to have largely resulted from innate immune and cellular immune responses to the vector (2124). Vector manufacturing and purification methods, a relatively unattributed factor, could also be a contributor to adverse effects $(25,26)$. Thus, it is now more important than ever to understand the mechanisms of immune activation against AAV to improve the safety of these gene therapy approaches $(17,18,25)$.

Most AAV vector biology studies have concentrated on dissecting the mechanisms of adaptive immune response to rAAVs. This bias stemmed from an early report showing that AAV2 vectors conferred minimal and transient activation of innate immunity, in contrast to the potent and prolonged adaptive responses elicited by adenovirus vectors (27). Thus, it was presumed that innate immunity against rAAVs is inconsequential. However, the innate immune system is the first line of defense against foreign pathogens and provides activation signals that are critical for adaptive immunity (28). Unfortunately, little is known about innate immunogenicity to rAAVs in humans. Here, we will briefly review the innate immune pathways that are implicated as being stimulated by rAAVs, and discuss the mechanisms that are known to be, or might be, activated in response to $\mathrm{rAAV}$ infection and subsequent suppression of transgene expression. We will also present selected strategies that show promise for overcoming the innate immune barriers to human gene therapy.

\section{Sensing of AAV vector elements}

Recognition of foreign viral particles by the innate immune response is achieved through continuous monitoring for structural motifs that are unique to non-self organisms, called pathogen-associated molecular patterns (PAMPs). Monitoring is carried out by immune receptors called pattern recognition receptors (PRRs). PRRs are expressed at high levels by innate immune cells, such as macrophages and dendritic cells (DCs). They can recognize microbial products on the cell surface, within the phagolysosome, in cytoplasmic compartments, and in the nucleus. PRR-mediated detection of AAV vector components and products (capsid, genome, transcript, etc.) is understudied. Figure 1 depicts the known and implicated innate immune pathways that rAAVs may activate upon infection. Related factors involved in the underlying mechanisms solved through other in vivo and in vitro model systems are also shown.

Based on protein domain homology, PRRs have been divided into several families: TLRs, DNA sensors such as cyclic GMP-AMP synthase (cGAS), nucleotide-binding and oligomerization domain- like (NOD-like) receptors (NLRs), C-type lectin receptors (CLRs), absent in melanoma 2-like (AIM2-like) receptors (ALRs), and retinoic acid-inducible gene I-like (RIG-I-like) receptors (RLRs) (29). These PRRs recognize and sense diverse PAMPs intrinsic to viruses, bacteria, fungi, and protozoa that include lipoproteins, carbohydrates, lipopolysaccharides, and nucleic acids. PRRs also recognize endogenous damage-associated molecular patterns (DAMPs), which, when gone awry, disrupt homeostasis and cause autoimmune diseases (30). Sensing of viral PAMPs triggers PRR-mediated intracellular signaling cascades via adaptor proteins, such as MyD88, MAVS, or STING. These signals lead to the expression of host defense genes, like the MHC genes, proinflammatory cytokines, chemokines, and type I and III IFNs (31). Transcriptionindependent cellular processes such as phagocytosis, autophagy, metabolism, cell death, and inflammasome/cytokine activation are also induced (32). Secreted IFNs and cytokines enhance innate immune responses via autocrine and paracrine mechanisms and induce expression of IFN-stimulated genes (ISGs) that inhibit viral replication and spread. Secreted cytokines and chemokines are also critical for inducing effective adaptive and memory immune responses (33). Based on their localization, PRRs can also be classified into 2 main classes: membrane-bound receptors (like TLRs) and intracellular receptors (like ALRs, NLRs, and cGAS).

\section{Membrane-bound DNA sensors: TLRs}

Membrane-bound receptors are found either on the cell surface or associated with endocytic compartments. They survey the extracellular space and within endosomes for the presence of microbial ligands. The most likely mode of AAV vector sensing is through TLR-mediated pattern recognition, which can trigger an innate immune response and promote activation of adaptive immunity (34). TLRs are single-pass transmembrane proteins and are characterized by a transmembrane domain, an intracellular Toll/IL-1R homology (TIR) domain, and an extracellular domain that binds to the corresponding PAMP. Humans and mice harbor 10 and 12 known TLRs, respectively. TLRs 1-9 are common to both species, TLR10 is expressed only in humans, and TLR11-TLR13 are exclusive to mice (35-37). Because of these differences, preclinical investigation in mice may not fully recapitulate how humans may respond to gene therapy vectors. TLR1, 2, 4, 5, 6, and 10 (glycoprotein-recognizing receptors) are commonly located on the cell surface, whereas TLR3, 7, 8, and 9 (nucleic acid-specific receptors) are generally found on the endosomal membrane (38). TLRs that have been implicated in initiating inflammatory responses to viruses are TLR2 (glycoproteins and lipoproteins), TLR3 (dsRNA), TLR4 (glycoproteins and bacterial LPS), TLR7 (ssRNA), TLR8 (ssRNA), and TLR9 (unmethylated CpG [cytosine-phosphate-guanine] DNA) (39). TLRs are mostly found in immune cells, including DCs, macrophages, B cells, and some T cells, but are also found in some nonimmune cells, such as fibroblasts and epithelial cells (40).

Receptor engagement with PAMPs or DAMPs leads to the formation of M-shaped TLR dimers or multimers, which in turn causes the multimerization of cytoplasmic TIR domains that recruit downstream adaptors (41). With the exception of TLR3, TLRs recruit the signaling adaptor MyD88, which mediates the phosphorylation of IRAK4 and IRAK1 (Figure 1). As illustrated in 


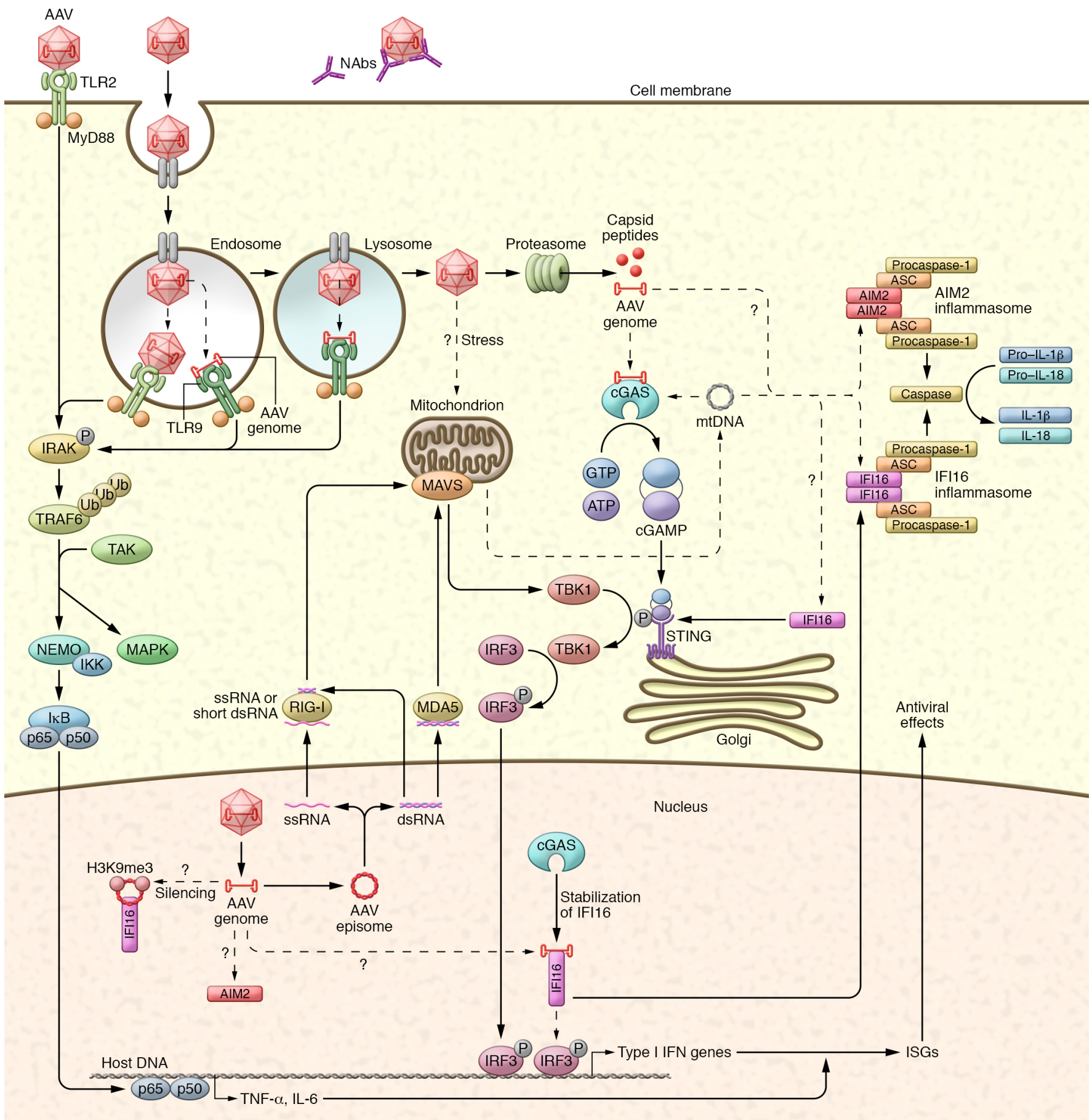

Figure 1. Detection of AAV vector elements by PRRs. rAAV capsids can activate TLR2 on the cell surface or endosomal membrane, which subsequently recruits MyD88 and phosphorylates IRAKs. rAAV capsids may break open in the endosome or lysosome and expose the genome to TLR9. Upon binding DNA, TLR9 activates the MyD88/IRAK pathway to induce proinflammatory cytokines like TNF- $\alpha$ and IL-6 (38). Vector genomes may also become exposed within the cytosol. Alternatively, rAAV-mediated stress may release mitochondrial DNA (mtDNA) to activate cytosolic DNA sensors. Upon binding DNA, cGAS promotes cGAMP synthesis, which activates the STING/IRF3 pathway and upregulates type I IFNs (146). IFNs and cytokines induce expression of ISGs and antiviral responses. IFI16 can promote cGAS-mediated production of cGAMP to activate STING. Upon binding DNA, AIM2 and IFI16 form the inflammasome and promote maturation of IL-1 $\beta$ and IL-18 (82). Nuclear-localized rAAV genomes may also activate cCAS and IFI16. Nuclear cGAS can induce IFN- $\beta$ production by stabilizing IFI16 (84), which can be exported into the cytoplasm to activate the inflammasome pathway (147). IFI16 also silences viral gene expression in the nucleus (76). AIM2 is also found in the nucleus (85), but it is not known whether AIM2 can sense viral DNA in nuclei. The cytosolic RNA sensors RIG-I and MDA5 can recognize RNA transcripts from rAAV and activate the TBK/IRF pathway to induce IFNs (88, 89, 92). Pathways that are only speculated to be involved are indicated by question marks. Pathways only implicated by circumstantial evidence are indicated with dashed arrows. 
Table 1. Strategies to overcome AAV genome sensing

Prevention strategy

Depleting the vector genome of $\mathrm{CpCs}$

TLR-inhibitory sequences

Hydroxychloroquine (HCQ)

Blocking reverse-strand transcription

\section{Proposed or demonstrated methods}

- CpG-depleted AAVrh32.33 vectors injected in the muscle lead to persistent transgene expression accompanied by reduced infiltration of effector T cells (48).

- Codon optimization to generate CpG-free $h$ F.IX vectors was used for hemophilia trials, but the conclusions were not definitive $(148,149)$.

- TLR9-inhibitory DNA sequences derived from human telomeres and composed of multiple copies of the TTAGGG motif are incorporated into the rAAV genome. This approach was reported to reduce rAAV-associated immune responses in mice and pigs $(60,61)$.

- The ability of TTAGGG motifs to inhibit CGAS and AIM2 inflammasome activation has also been documented $(86,87)$.

- The antimalarial agent HCQ has also shown immunomodulatory effects. Sequestration and accumulation of HCQ within the lysosomal compartment hinders intraorganellar processes, like nucleic acid assembly and the ability of TLR9 to bind DNA (150-152).

- HCQ administration 1 hour before rAAV treatment has been shown to increase transduction efficiency in both murine and human tissues, and within RPE and photoreceptor cells in retinal explants (77).

- HCQ inhibits binding of DNA not only to TLR9, but also to CGAS (77).

- Engineering ITRs that lack promoter function or have weak promoter function may decrease the formation of dsRNAs.

- Engineering the vector by mutating the transcription initiation site at the 3'-ITR to block transcription may decrease the formation of dsRNAs (90).

- Several viruses, like hepatitis C virus, Sendai virus, and herpes simplex virus 1 (HSV1), actively inhibit RIG-I function. Expression of factors like hepatic selenoprotein (94), RNA helicase SKIV2L (95), or the long noncoding RNA IncATV (96) are increased upon viral infection, leading to the suppression of RIG-I activation. Investigation into how viruses suppress RLR activation may uncover novel approaches that prevent long-term immunity in AAV-transduced cells.
Figure 1, activated IRAKs lead to transcription of several proinflammatory cytokines, including TNF- $\alpha$ and IL-6 (35). Conversely, TLR3 uses TRIF as the adaptor molecule for TRAF6-mediated activation of TAK1 and production of inflammatory cytokines. Additionally, TLR3 and TLR4 are capable of employing TRIF to induce transcription of type I IFNs through a pathway involving TANK-binding kinase-1 (TBK1) and interferon regulatory factor 3 (IRF3), which contribute to the antiviral inflammatory response $(42,43)$. TLR7, 8 , and 9 can also induce type I IFNs by employing the MyD88 signaling pathway (44).

Innate immunity against rAAVs is remarkably muted compared with that against adenoviruses, because rAAVs seem to lack the inflammatory cues to confer an effective CTL response against the transduced cell $(27,45)$. TLR9, however, plays a vital role in shaping immune responses to both transgene and capsid. TLR9 binds specifically to unmethylated CpG dinucleotides, which are present in the genomes of bacteria and DNA viruses. TLR9-mediated sensing of CpG motifs within rAAV genomes may occur during endosomal trafficking, where virions with partially exposed genomes are recognized (Figure 1) (46). Alternatively, degradation of the viral capsid in the lysosome can expose the genome to TLR9, which then signals through MyD88 to activate $\mathrm{NF}-\kappa \mathrm{B}$ and IRF7 to regulate type I IFNs and ISGs (Figure 1) (47). Induction of type I IFNs through TLR9 and MyD88 signaling was shown to be transgene- and serotype-independent (48). In this work, rAAV-delivered transgene expression was eliminated in WT mice but not in TLR9-knockout animals. In addition, when the transgene was depleted of $\mathrm{CpG}$ dinucleotides, expression was retained for more than 90 days, while vectors carrying unmodified transgenes conferred a loss of expression after 30 days (48). Studies in mice have also revealed that while antibody responses to the transgene were mostly independent of the innate immune sensors investigated, the TLR9/MyD88 pathway was critical for priming a $\mathrm{CD} 8^{+} \mathrm{T}$ cell response to AAV capsid and the transgene product following intramuscular injection of vector $(34,47,49-51)$.
Notably, use of self-complementary AAV (scAAV) vectors was shown to increase vector potency by providing notably faster and stronger transgene expression, allowing for lower and possibly safer vector doses (52). However, this difference in genome configuration potentially influences the innate response as well. The use of scAAV genomes was revealed to increase the innate immune response to the transgene compared with single-stranded vectors $(53,54)$. This increase was also attributed to an amplified activation of the TLR9/MyD88 pathway (34).

Apart from TLR9 activation, increased transcription of TLR2, which recognizes microbial protein and glycolipid structures, was also observed upon rAAV infection and is consistent with reports demonstrating its role in sensing non-enveloped viral particles (55-57). This result raised the possibility of TLR2's involvement in innate immunity against rAAVs (53). In subsequent studies, human cells were shown to sense AAV capsid through TLR2 (Figure 1) (58). Finally, MyD88, a B cell-intrinsic downstream mediator of TLR2 and TLR9 signaling, has been suggested to be pivotal in the formation of Th1-dependent antibodies to AAV (59).

Several strategies to overcome TLR signaling are summarized in Table 1. As mentioned above, preventing TLR9 signaling by depleting $\mathrm{CpG}$ dinucleotides in the vector genome has been shown to enhance transgene expression and reduce infiltration of effector T cells in the muscle (48). Incorporation of TLR9inhibitory (TLR9i) sequences has recently been reported to reduce rAAV-associated immune responses in mice and pigs $(60,61)$.

\section{Detection of rAAV genomes by cytosolic DNA sensors}

Several cytosolic DNA sensors are known to detect viral DNA (62), including cGAS (63), IFN-inducible protein 16 (IFI16) (64), and AIM2 (65) (summarized in Figure 1). They bind DNA in a sequence-independent but length- and structure-dependent manner. cGAS binds double-stranded DNA (dsDNA) or DNARNA hybrids that are preferentially longer than 36 bp (66-69). 
It dimerizes upon binding to DNA and triggers a cascade involving STING and TBK1 to induce the transcription of type I IFN genes and antiviral cytokines, such as TNF- $\alpha$ and IL-6 (70). Mice deficient in cGAS and STING are more susceptible to lethal herpes simplex virus 1 (HSV1) infection because of a decrease in type I IFN response $(71,72)$. IFI16 is a member of the pyrin and HIN (hematopoietic IFN-inducible nuclear) domain-containing (PYHIN) family of proteins (65). It has emerged as a prominent DNA sensor of HSVs (73). IFI16 exists in both the cytosol and the nucleus and is activated by both single-stranded DNA and dsDNA (64). The optimal length of DNA that serves as a ligand for IFI16 is $70 \mathrm{bp}$ (74). IFI16 may cooperate with the cGAS/STING pathway in some contexts $(64,75)$. IFI16 also silences viral gene expression by facilitating heterochromatinization of the viral genome in the nucleus (76). AIM2, another PYHIN family member, binds to DNA stretches about 80 bp in length (74). AIM2 can bind dsDNA in the cytoplasm and forms an inflammasome with ASC, activates caspase-1, and produces mature IL-1 $\beta$ and IL-18. Knockdown of AIM2 inhibits the activation of caspase- 1 in response to the presence of viral dsDNA (65).

The AAV genome contains elements that can potentially activate cytosolic DNA sensors, such as the ITR hairpin structures. A recent study showed that $\mathrm{rAAV}$ induces the expression of DNA sensors including cGAS and antiviral genes, such as TNF- $\alpha$ and IFN- $\gamma$ (77). In this study, rAAV transduction was 6-fold higher in $\mathrm{cGAS}^{-/-}$mouse embryonic fibroblasts than in WT fibroblasts. How rAAV activates DNA sensors is not completely known. The dogma is that the $\mathrm{rAAV}$ genome is encapsidated until the virion arrives in the nucleus, where the genome is then released. One hypothesis is that proteasomal degradation of the capsid exposes the AAV genome (78). Another possibility is that AAV infection stresses the cell to release mitochondrial DNA into the cytosol, which then activates the cytosolic DNA sensors (Figure 1) $(79,80)$. Alternatively, DNA sensors may detect the AAV genome in the nucleus. IFI16 can shuttle between the nucleus and cytoplasm and recognizes viral DNA in the nucleus by scanning along DNA duplexes (Figure 1) (81). Upon binding to viral DNA, IFI16 can move to the cytosol to activate the inflammasome pathway $(74,82,83)$. IFI16 is stabilized by nuclear cGAS and induces IFN- $\beta$ production through an unknown mechanism (Figure 1) (84). Although AIM2 is known to sense DNA damage in the nucleus and induces inflammasome activation (85), whether AIM2 can sense viral DNA in the nucleus is unknown. Further studies are required to elucidate whether AAV infection activates the AIM2 inflammasome pathway.

Strategies to overcome cytosolic DNA sensors have not been heavily explored (Table 1), since their roles for limiting rAAV efficacy are less established. The ability of TLR9i motifs and hydroxychloroquine (HCQ) to inhibit cGAS and AIM2 inflammasome activation has been documented $(77,86,87)$. HCQ administration 1 hour before rAAV treatment has been shown to increase transduction efficiency in both murine and human tissues, and within retinal pigment epithelial and photoreceptor cells in retinal explants (77).

\section{Innate immunity against transgene transcripts: RNA sensors}

Cytosolic RIG-I-like receptors (RLRs) are expressed in almost all mammalian cell types. As the main family of cytosolic RNA sensors, RLRs play key roles in virus recognition $(88,89)$. The members of this family include the prototypic RIG-I, MDA5, and LGP2. RIG-I and MDA5 have similar domain structures: two tandem caspase activation and recruitment domains (2CARDs) at the $\mathrm{N}$-terminus, a central DExH-box helicase domain, and a C-terminal regulatory domain. RIG-I recognizes 5 '-triphosphorylated blunt-ended short dsRNA or single-stranded RNA hairpins of positive- and negative-strand viruses. MDA5, in contrast, preferentially recognizes long dsRNA and binds to the RNA backbone of dsRNA viruses or dsRNA replication intermediates of positivestrand RNA viruses. LGP2 has no signaling activity, because it lacks N-terminal 2CARDs, but is able to regulate RIG-I and MDA5 signaling via its ability to bind RNA $(88,89)$. MDA5 and RIG-I have a common signaling adaptor, MAVS, which induces type I IFN production upon virus infection (Figure 1).

Although DNA-sensing molecules mediate innate immunity against AAVs, a dsRNA-mediated response may contribute to the therapeutic failure at later stages of vector transduction. The AAV ITR has inherent promoter activity $(90,91)$. Therefore, the presence of $5^{\prime}$ - and $3^{\prime}$-ITRs in rAAV genomes may result in the production of both sense and antisense RNAs that can form dsRNA intermediates in the cytosol. These dsRNA molecules are subject to recognition by MDA5 and RIG-I, leading to production of IFN- $\beta$ (92). Similarly, RIG-I and MDA5 were also found to be upregulated in the primate retina after long-term AAV transduction (93).

As with other cytosolic sensors, the impact of RLRs on rAAV is still under investigation. Several viruses, like hepatitis $C$ virus, Sendai virus, and HSV1, actively inhibit RIG-I function. Expression of factors like hepatic selenoprotein (94), RNA helicase SKIV2L (95), or the long noncoding RNA lncATV (96) is increased upon viral infection, leading to the suppression of RIG-I activation. Investigation into how viruses suppress RLR activation may uncover novel approaches that prevent long-term immunity in AAV-transduced cells. Additional potential approaches are described in Table 1.

\section{Capsid immunity and the complement system}

The rAAV protein capsid can encounter multiple aspects of the host's immune system at different stages, posing considerable barriers to effective gene delivery and long-term gene expression. Capsid-based inflammatory response, as noted earlier, is potentially mediated by TLR2 (Figure 1). However, the principal concern with respect to immunity against the AAV capsid are preexisting NAbs. Notably, empty AAV capsids on their own can induce innate immune responses $(58,97)$. A substantial portion of the human population develop anti-AAV NAbs against naturally circulating AAVs, which can be detected in children as young as 2 years of age $(7,8,98,99)$. NAbs are typically characterized by broad cross-reactivity across different serotypes. NAb-mediated reduction in vector transduction was first documented in a factor IX (AAV2-F.IX) liver gene transfer trial (11). Two individuals in the cohort who were given a high vector dose had NAb titers of $1: 2$ and 1:17. As a consequence, they displayed extremely low circulating F.IX levels upon vector treatment (11). NHPs, which are natural hosts for AAV8, lack the capacity to be transduced by high doses of AAV8-F.IX when NAb titers are as low as 1:5 (100). NAb titers are predicted to reduce patient inclusion for rare disease therapies by as much as $50 \%$ (7). Fortunately, gene therapy via direct CNS 


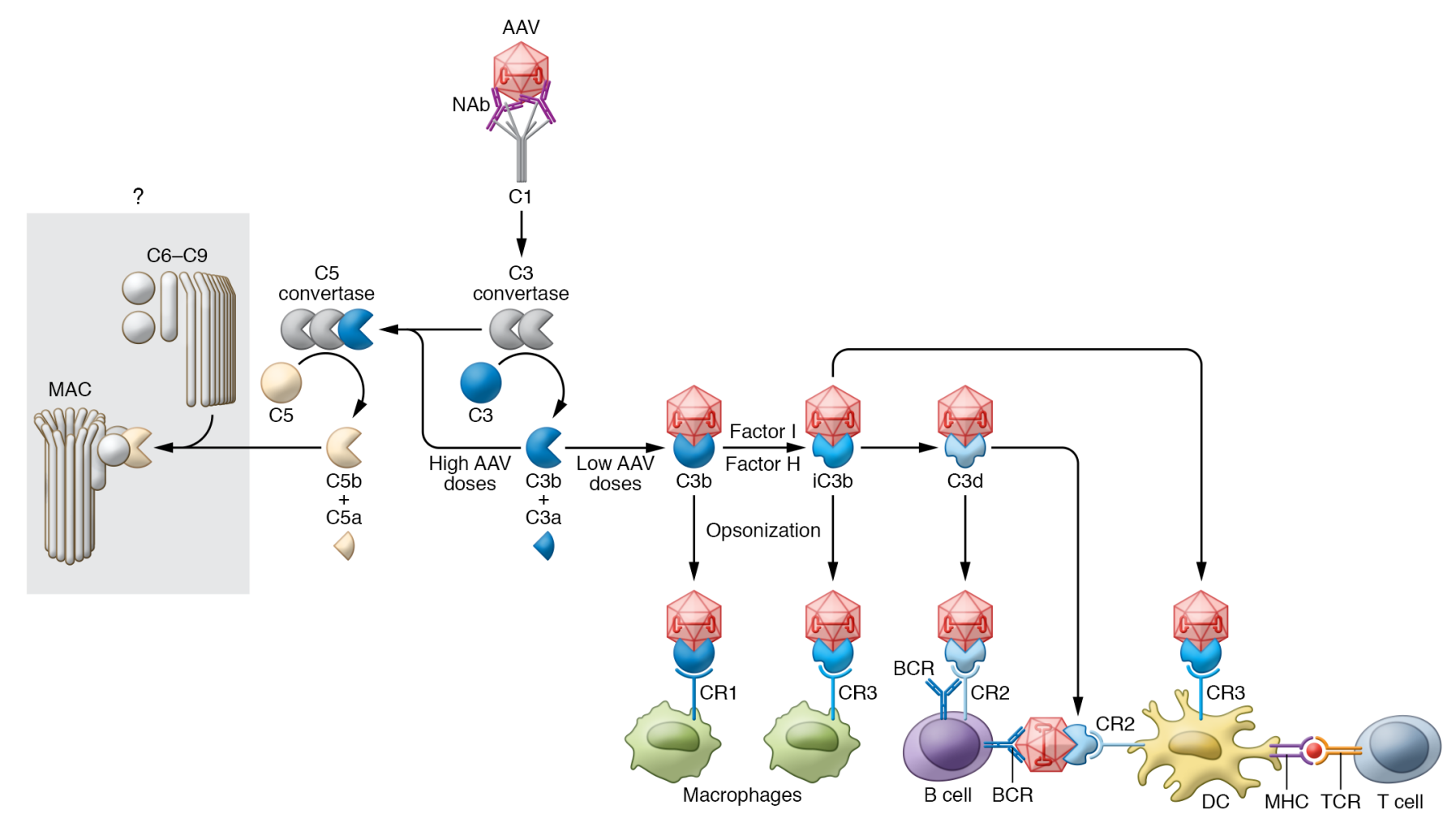

Figure 2. Complement activation by AAVs. Antibodies bound to AAV particles are recognized by the complement protein $\mathrm{C} 1$ complex. When high doses of AAV are administered, AAV-antibody complex activates the classical pathway of complement, eventually leading to the formation of the membrane attack complex (MAC) (105). The target of the MAC ring during AAV infection is unclear. When low AAV doses are administered, C3b can bind to the AAV capsid, where it is converted to iC $3 \mathrm{~b}$ and subsequently to C3d by factor I and other cofactors. Cleavage fragments of C3 opsonize the target structure and serve as bridging molecules with receptors on the surface of the phagocytes. CR1 and CR3 expressed on the macrophage surface interact with C3b- or iC3b-opsonized AAV particles, leading to phagocytosis and macrophage activation. CR3 interaction with iC3b-opsonized AAV virions on DC surfaces also results in endocytosis and antigen presentation to naive T cells. C3d-bound AAVs can be recognized by CR2 on B cell surfaces. Co-ligation of CR2 with B cell receptor (BCR) results in augmented signaling that effectively lowers the threshold for B cell clonal expansion. Alternatively, DCs can also trap the C3d-opsonized $A A V$ via CR2 and present the antigen to naive or previously antigen-engaged B cells during the processes of affinity maturation, isotype switching, and the generation of effector and memory B cells.

or eye delivery is reasonably well tolerated in patients with NAbs, since the brain and the eye are considered immune-privileged organs, though they are still not completely shielded from circulating NAbs (101-104). The presence of NAbs is bound to profoundly impact the efficacy of AAV-mediated gene transfer and should be measured carefully before enrollment of prospective subjects.

In addition to causing a lack of therapeutic efficacy, the presence of capsid NAbs can also trigger complement activation, which at high doses may become a safety concern. The complement system comprises over 30 fluid-phase proteins and several membrane-bound proteins that are an essential component of the host innate immune system. The main site of synthesis for most complement proteins is the liver. Several other tissues also produce various complement components. Soluble complement components are distributed throughout the body's tissues and fluids. Many key activation components of complement exist as inactive precursors and undergo a cascade of proteolytic cleavage events and activation steps to generate the final products of the complement system (Figure 2) (105).

Depending on the pathogenic context, the complement system cascade is initiated by three different pathways - classical, lectin, and alternative - all of which converge at the level of com- plement protein $\mathrm{C} 3$ and lead to the formation of the membrane attack complex (MAC; terminal pathway). The classical pathway is activated upon recognition of antigen-antibody immune complexes by the C1 complex. The lectin pathway is activated upon recognition of sugars on pathogen surfaces by mannose-binding lectins (MBLs). Under normal physiological conditions, the alternative pathway is constitutively active at low levels and serves as a surveillance system to remove invading pathogens before the development of adaptive responses. All pathways cleave their precursor factors and converge at $\mathrm{C} 3$ convertase, which cleaves C3 into functional fragments, C3a and C3b. As illustrated in Figure 2, C3b acts as an opsonin or cleaves $\mathrm{C} 5$ to initiate a cascade to form MAC, which is composed of C5b to C9, on the surface of a pathogen or pathogen-infected cell for lysis (105). Opsonization of pathogens marks their removal by phagocytes and cell lysis. This action also serves as a link between innate and adaptive immunity (106). Antigens coated with complement activation fragments, like C3dg, assist in initiating a powerful costimulatory signal by ligating the $\mathrm{B}$ cell receptor to complement receptor 2 (CR2) on B cells, thereby reducing the threshold of B cell receptor activation and increasing the amplitude of the antibody response (107). Furthermore, C3a and C5a are involved in main- 


\section{Table 2. Strategies to overcome capsid immunity}

Prevention strategy

Pharmacological agents

Nonspecific cleaving of circulating lgGs

Plasmapheresis

Epitope masking

Structural modifications

Directed evolution

\section{Proposed or demonstrated methods}

- Rituximab is an anti-CD20 mAb that leads to B cell depletion (9).

- Rapamycin is a macrolide compound that inhibits T and B cell activation by reducing their sensitivity to IL-2 via mTOR inhibition (153).

- Rituximab and rapamycin administration, along with serotype switching (154), has also been demonstrated to induce immune tolerance and allows repeated rAAV administrations (155-158).

- Treatment with IdeS, a cysteine protease derived from Streptococcus pyogenes (and its homolog IdeZ, which was identified from S. equi ssp. zooepidemicus), may provide complete, rapid, and transient NAb-free windows for AAV vector delivery to patients $(127,128)$.

- Removal of large-molecular weight molecules, like antibodies from the blood, can ensure that there is minimal contact between the vector and NAbs. The technique has been used for successful and sustained gene transfer in NHPs and humans $(122,123)$.

- AAV-specific plasmapheresis column was used to selectively and effectively deplete anti-AAV NAbs without affecting the total immunoglobulin pool from the plasma in NHPs (124).

- Coating the AAV surface with lipids or cell-derived extracellular vesicles to mask AAV epitopes from NAbs can prevent neutralization of vector particles (125).

- Exosome-encapsulated AAVs (exo-AAV5 and exo-AAV8) have been shown to increase hF.IX expression 10-fold, along with a corresponding increase in transduction efficiency (126).

- Structural studies have proposed that NAb recognition sites are localized in specific areas of the AAV capsid (115). Modifying these regions may yield AAV mutants that have the ability to evade NAb recognition without affecting transduction efficiency or tissue tropism (116).

- Capsid residue 265 within VP1 of AAV2 was mutated to create AAV2.5. This change resulted in a capsid that was not recognized by mAb A2O (antibody that binds AAV2 and AAV3 capsids) (117), leading to enhanced transduction of skeletal muscle (118).

- Directed evolution can be used to identify AAV variants with decreased sensitivity to NAbs by iterative rounds of mutational screening to evolve AAV variants under selection pressure (9). This is achieved either by error-prone PCR to generate a library with random mutations or by creation of a DNA-shuffled library using preexisting serotypes as templates (119).

- AAV-DJ was generated from 8 serotypes in primary human hepatocytes in the presence of pooled human intravenous immunoglobulin (IVIG) (120). This non-natural capsid is composed of residues from AAV2, AAV8, and AAV9 serotypes and transduces liver more efficiently in mice injected with IVIG as compared with its parental capsid.

- Variants have also been isolated from humanized mouse models. These mutants escape NAb recognition in human sera and can transduce human hepatocytes more efficiently than preexisting AAV serotypes (121). The effectiveness of these capsids still needs to be tested in clinical settings.

Alternative strategies

can bypass encountering NAbs in circulation (125).

- Saline flushing with or without balloon catheter isolation in hepatic circulation also minimized the inhibitory effect of NAbs (159).

- Use of less seroprevalent capsids may evade detection by NAbs (160).

- Using empty capsids as decoys also helps in reducing the functionally effective levels of NAbs in circulation. Moreover, because empty capsids are not immunologically inert, mutant empty capsids could be used to reduce the effects of undesired immune activation (129).

taining $\mathrm{T}$ cell viability, proliferation, and differentiation as illustrated in Figure 2 (108).

Over the years, there has been scant evidence of AAV-mediated complement activation, and the commonly held belief was that the complement system does not play a considerable role in innate response to AAVs. It is also noteworthy that there are no currently published large-animal data to support complement activation by immune complex formation. Complement activation in humans upon administration of high doses of rAAV is also based on the available pathological evidence, and hence some of the mechanisms proposed in this section are speculative. Nonetheless, recent in vitro studies showed that the viral capsid interacts with C3 fragments, leading to enhanced uptake of AAV2 vectors by macrophages (109). This uptake was abrogated by heatinactivation or in C3-depleted mouse/human serum. Immunoprecipitation studies also confirmed that the AAV capsid binds to the complement protein $\mathrm{iC} 3 \mathrm{~b}$ and the complement-inhibitory protein factor $\mathrm{H}(\mathrm{FH})$ in serum. C3b is generated by $\mathrm{C} 3$ activation through the alternative or the classical pathway. C3b is then deposited on viral surfaces and has two possible fates: (i) $\mathrm{C} 3 \mathrm{~b}$ can then recruit factor I (FI) in the presence of a membrane-bound (e.g., CD46 or $\mathrm{CR} 1$ ) or a soluble (e.g., FH) cofactor that rapidly converts $\mathrm{C} 3 \mathrm{~b}$ to
iC3b, which in turn limits downstream complement activation. (ii) Alternatively, $\mathrm{C} 3 \mathrm{~b}$ can recruit factor $\mathrm{B}$, which, in the absence of a cofactor and FI, results in the formation of the $\mathrm{C} 3$ convertase and complement activation (106). Many pathogens have evolved mechanisms to evade direct activation of the complement system by mimicking host surfaces, thereby recruiting $\mathrm{FH}$ (110). At lower doses, AAV associates with both FH and iC3b, which suggests that AAVs actively inhibit the complement cascade (Figure 2). Although complement activation can be avoided, conversion of $\mathrm{AAV}$-bound C $3 \mathrm{~b}$ to iC $3 \mathrm{~b}$ might serve as an opsonin to increase AAV uptake by macrophages. This possibility may explain why treatments with AAV1, AAV2, and AAV8 vectors are associated with increased macrophage activation and a corresponding increase in the activation of cytokines, like macrophage inflammatory protein

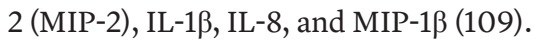

Interestingly, higher doses of AAV significantly activate complement over baseline - a response that can be blocked by EGTA, which chelates $\mathrm{Ca}^{2+}$ and is essential for classical and lectin pathway activation. This finding strongly suggests that complement activation by AAV may be primarily antibody-dependent (Figure 2) (109). Indeed, involvement of the classical pathway was confirmed by the loss of complement activation in IgG-depleted 
Table 3. Strategies to overcome complement activation

Prevention strategy

Systemic complement inhibition

Neutralizing antibody reduction

\section{Proposed or demonstrated methods}

- Eculizumab is an mAb that inhibits component $[5$ and prevents MAC formation. It is approved for the treatment of rare disorders involving complement hyperactivation, particularly paroxysmal nocturnal hemoglobinuria (PNH), atypical hemolytic uremic syndrome, and refractory generalized myasthenia gravis (161).

- Therapeutic interventions that block different molecules of the complement pathway, including naturally occurring human inhibitory proteins (e.g., FH or factor I), blocking antibodies, peptides, small-molecule inhibitors, aptamers, siRNAs, and antisense oligonucleotides, are being developed and have been attempted for the treatment of diseases like age-related macular degeneration, neuromyelitis optica, and PNH with mixed results (162).

- Since complement activation by AAVs takes place via the classical pathway and is triggered by antibody binding, approaches to reduce NAbs should also mitigate complement activation (109).

- Pharmacological agents like rapamycin and rituximab and encapsulating AAVs inside exosomes can be potential methods to minimize AAV interaction with antibodies, and hence eliminate complement activation.

Suppressing $[3$ activation
- One strategy being used by Apellis Pharmaceuticals is the use of APL-9 in conjunction with AAV administration. APL-9 is a PEGylated synthetic cyclic peptide that binds specifically to $C 3$ and prevents $[3$ activation, effectively blocking all 3 pathways of complement activation. APL-9 demonstrated control of complement within 1 hour of administration that lasted up to 12 hours. Multiple doses tested achieved complete suppression of the AH50 hemolytic activity. APL-9 was well tolerated with no serious adverse events reported (130). serum (109). Moreover, activation of innate immune-specific chemokines by AAV-complement complexes is not enhanced at these concentrations. Nevertheless, mice deficient in CR1/2 or C3 were less capable of mounting a humoral immune response to AAV than WT mice, indicating a role for complement in the production of AAV-specific antibodies. As discussed above, co-engagement of CR2 with the B cell antigen receptor enhances antibody production $(111,112)$. Additional evidence of complement activation in response to AAVs arose from clinical trials for Duchenne muscular dystrophy in humans. A few patients who were given high doses of rAAV in trials (ClinicalTrials.gov identifiers: NCT03362502 and NCT03368742) experienced transient and/or acute renal impairment, accompanied by activation of the complement system $(113,114)$.

Multiple strategies to overcome capsid immunity and evasion of NAbs have been developed throughout the years. Structural modification of NAb recognition sites and directed evolution to generate novel AAV capsids have also been found to be effective strategies to evade NAbs in preclinical studies $(9,115-121)$. Additional strategies that have proven effective in mice and NHPs include plasmapheresis, rAAV epitope masking, use of IgGdegrading enzymes, and injection of empty AAV capsids as decoys (122-129). Additional promising strategies are described in Table 2.

Strategies to directly overcome complement activation have been widely developed. These modalities are now being tested in the context of AAV-based gene therapies (Table 3). One of the more promising strategies is the use of $\mathrm{C} 3$ modulator drugs, such as APL9. APL-9 prevents C3 activation, effectively blocking all three pathways of complement activation. APL-9, when used in conjunction with AAV administration, demonstrated control of complement within 1 hour of administration that lasted up to 12 hours (130).

\section{Cell types contributing to innate immunity}

There is limited literature on the types of cells that are involved in mounting an innate immune response to AAVs. It is known that PRRs like cGAS are expressed in all cell types but are particularly enriched in professional antigen-presenting cells (APCs). TLRs, especially TLR9, are restricted to B cells and plasmacytoid DCs
(pDCs) in humans. Other studies have shown that anti-AAV capsid IgG2 antibody production is not dependent on any specific TLR alone, but on intrinsic MyD88 signaling in B cells, and not T cells $(34,59)$. A possible explanation for this observation is that stimulation of the TLR9/MyD88 pathway, particularly in monocyte-derived DCs, may drive differentiation of follicular Th cells and promote robust IgG2c production by B cells/plasma cells. However, IgG1 and IgM antibodies were generated independent of MyD88 activation, indicating differential kinetics. Since it is well established that $\mathrm{T}$ cell activation is critical for a robust humoral immune response, this could be attributed to a shift in activation of $\mathrm{Th} 2 \mathrm{CD}^{+} \mathrm{T}$ cells as opposed to a Th1 response and subsequent IgG subclass induction (33).

Intravenous administration of AAV was seen to induce transient expression of TNF- $\alpha$, RANTES (regulated on activation, normal $\mathrm{T}$ cell expressed, and secreted), IP-10, MIP-1 $\beta$, MCP1 , and MIP-2 mRNAs in the liver. These transcripts return to baseline levels after 6 hours. This activation was revealed to be dependent on Kupffer cells, the resident macrophages of the liver (27). Another study also showed that scAAV administration increased macrophage infiltration in the liver 6-fold, an effect that was reversed by administration of TLR9i oligonucleotides (53). Contrary to these findings, further studies demonstrated that innate immune recognition of AAV takes place in mouse or human pDCs, and not in conventional DCs (cDCs), Kupffer cells, or macrophages (131). Transient depletion of CD11c cells in mice significantly reduced $\mathrm{CD}^{+} \mathrm{T}$ cell response. However, inactivation of Kupffer cells and macrophages by the macrophage inhibitor $\mathrm{GdCl}_{3}$ did not affect the response (131).

Most reports that investigate MHC class I presentation of AAV capsid regard the target cells that are flagged for destruction by $C D 8^{+} \mathrm{T}$ cells. This phenomenon is presumably a consequence of cross-priming of $\mathrm{CD}^{+} \mathrm{T}$ cells from antigen presentation by APCs $(132,133)$. However, some studies have highlighted the vital participation of professional APCs in MHC class I-mediated presentation of antigens originating from the AAV capsid or the transgene in eliciting a $\mathrm{CD}^{+} \mathrm{T}$ cell response and activation of a humoral response. Initial in vitro data in DCs isolated from 
A

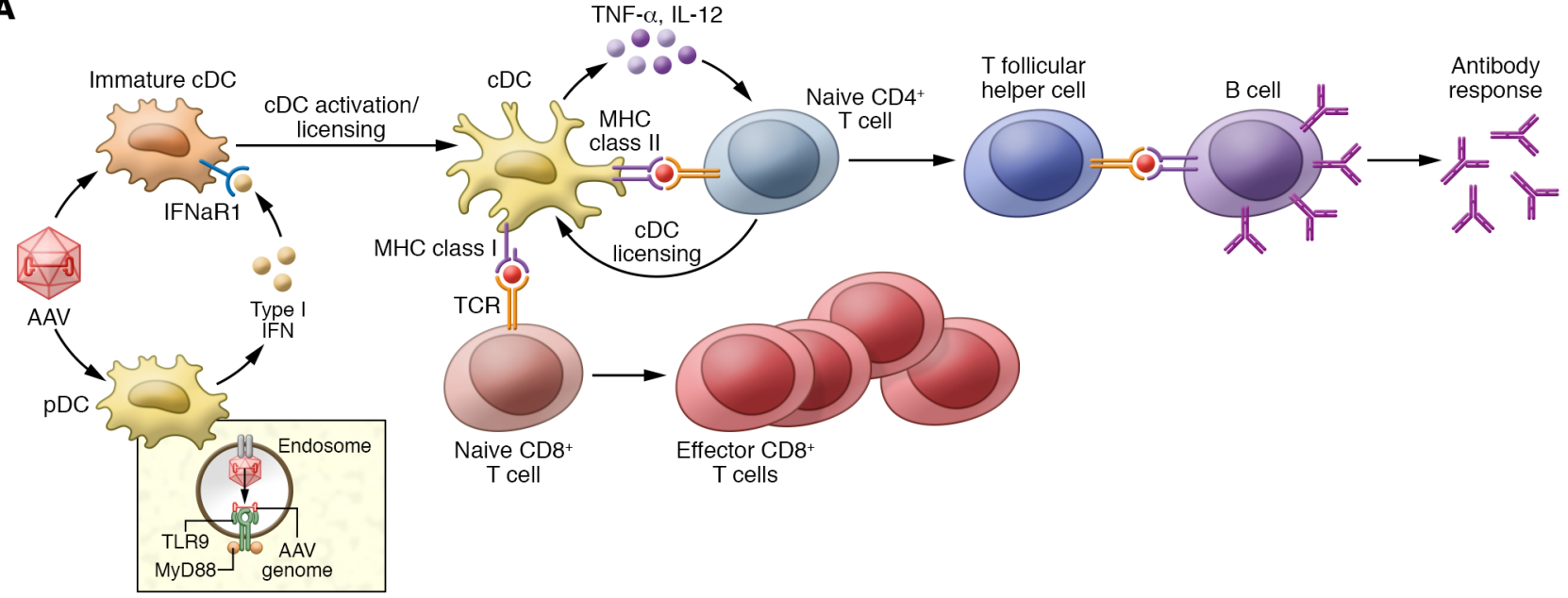

B

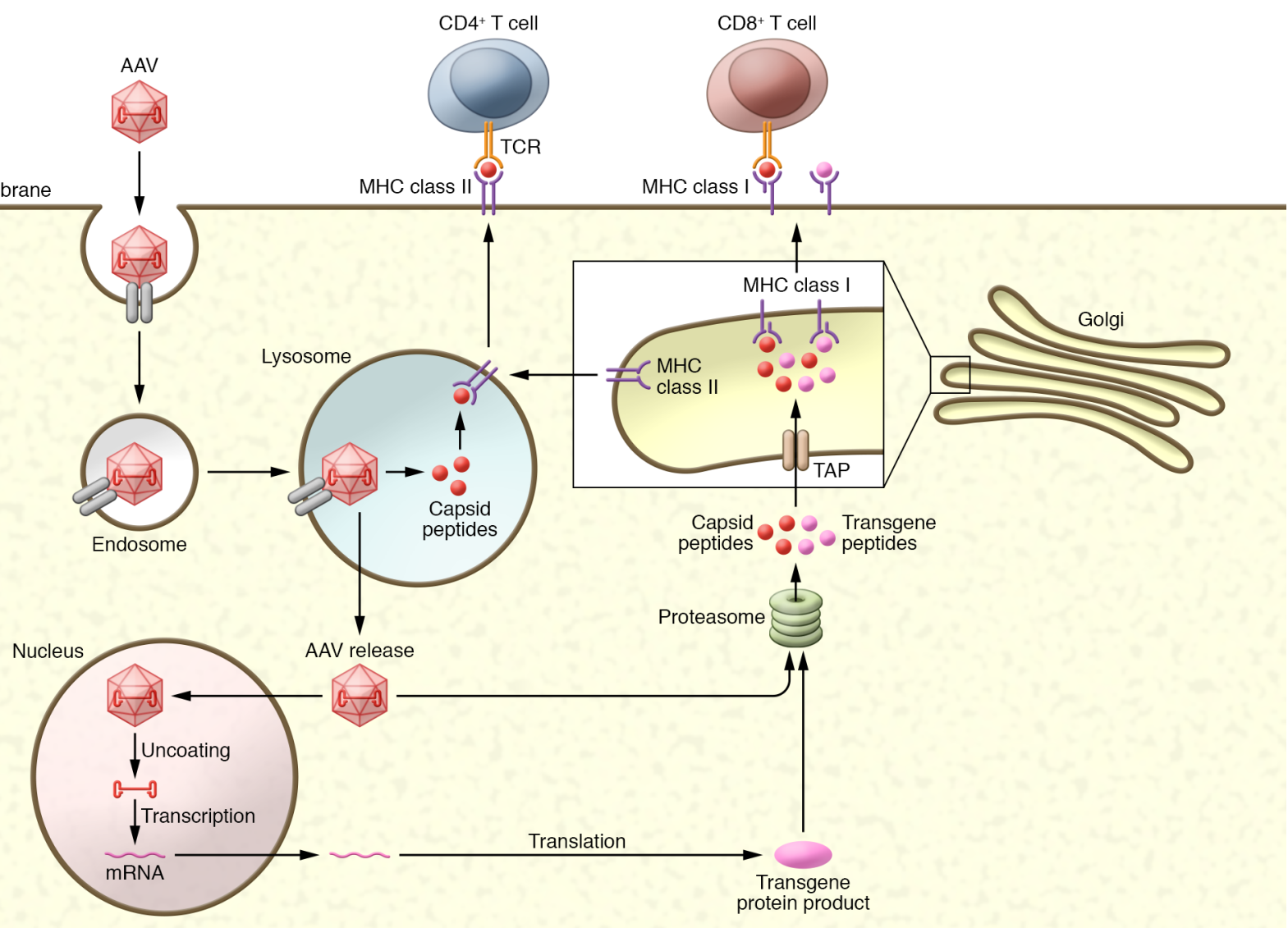

Figure 3. The role of APC-mediated immune responses toward AAV vectors. (A) The mechanism of DC activation by rAAV. Vector genome sensing by TLR9 in pDCs' endosomes triggers the activation of the TLR9/MyD88 signaling pathway that culminates in pDCs producing type I IFNs that directly signal to immature cDCs. This signaling event is required for effective priming and leads to activation and licensing of immature cDCs to mature cDCs. Licensing of $\mathrm{CDCs}$ enhances their ability to activate T cells. Activated $\mathrm{CDCs}$ also interact with $\mathrm{CD} 4^{+} \mathrm{T}$ cells, which may additionally contribute to licensing the $\mathrm{CDCs}$ to activate rAAV capsid-specific CD8 ${ }^{+} \mathrm{T}$ cells. Activated CD4+ Th cells also promote antibody formation against the rAAV capsid. (B) Depiction of rAAVspecific antigen presentation by APCs. Upon entry of AAV virions into cells by endocytosis, rAAV capsids can either be degraded in lysosomes or escape into the cytoplasm. Transcription and translation of the vector genome in the nucleus generate transgene proteins that, along with viral capsids, can be ubiquitylated and degraded in the proteasome into small peptides. These peptides are transported into the Golgi/endoplasmic reticulum by transporter associated with antigen presentation (TAP), loaded onto the MHC class I molecule, and presented on the surface of the target cell. This causes the cell to be recognized by a CD8 ${ }^{+}$cell and, finally, eliminated by a capsid-specific CTL response. Capsid peptides are also loaded onto MHC class II molecules for presentation to $\mathrm{CD}^{+} \mathrm{T}$ cells for subsequent $\mathrm{B}$ cell activation and antibody production. 
Table 4. Strategies targeting immune cell mechanisms

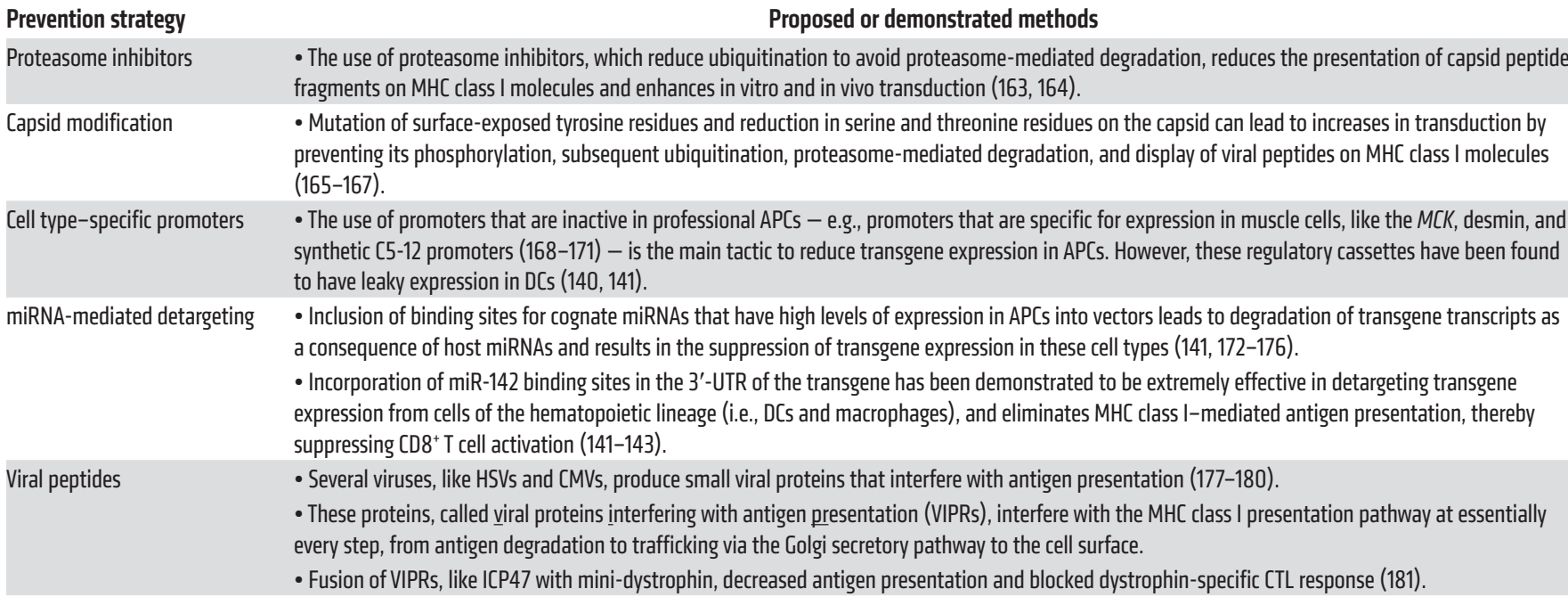

mice revealed that only pDCs were capable of producing type I IFNs in response to AAV, which in turn leads to a $\mathrm{CD}^{+} \mathrm{T}$ cell response (47). Later studies have painted a more complicated picture. It was observed that $\mathrm{pDCs}$ and $\mathrm{CDCs}$ need to cooperate to recognize the viral genome via a TLR, present the viral antigen, and activate a $\mathrm{CD}^{+} \mathrm{T}$ cell response (131). According to this report, pDCs carry out sensing of nucleic acids via TLR9 and produce large amounts of type I IFN, which acts as an activation signal for cDCs. This process is called "DC licensing" and involves the uptake and processing of viral particles by cDCs, followed by cross-presentation of AAV capsid proteins, via cross-priming of CD8 ${ }^{+} \mathrm{T}$ cells (Figure $3 \mathrm{~A}$ ) (131). The cross-presentation of AAV capsids on MHC class I is proteasome-dependent and is called the cytosolic pathway, which also leads to cross-priming of $\mathrm{CD} 8^{+} \mathrm{T}$ cells (Figure 3B) $(133,134)$. Antibody production against AAV capsids has also been observed (59). Capsid peptides are loaded onto MHC class II and presented to $\mathrm{CD} 4^{+} \mathrm{T}$ cells (Figure $\left.3 \mathrm{~B}\right)(6,135,136)$. This phenomenon was crucially discovered when patients treated with F.IX gene therapy (rAAV8-F.IX) showed an initial decrease in F.IX levels followed by a subsequent increase after an administration of steroids $(137,138)$. Studies revealed that the AAV-transduced hepatocytes were cleared by a capsid-specific CTL response. Additional studies in mice and humans indicated that the level of capsid-specific antigens presented on target cells determines the level of the CTL response (137-139).

A multitude of strategies to directly mute immune cell activation have been developed. The use of cell type-specific promoters and miRNA-mediated detargeting have been widely explored. Promoters - natural and synthetic - have been used for specific expression in muscle cells but have been found to have leaky expression in DCs $(140,141)$. Incorporation of miR-142 binding sites in the $3^{\prime}$-UTR of the transgene has been demonstrated to be extremely effective in detargeting transgene expression from APCs, thereby suppressing CD8 ${ }^{+} \mathrm{T}$ cell activation (141-143). Additional extensively used strategies are summarized in Table 4.

\section{Conclusions}

We have discussed the current obstacles that innate immunity poses for the successful implementation of rAAVs as reliable gene therapy medications. Although safety is the principal goal for all gene therapies, these therapies also represent the only treatment option for many rare and chronic genetic diseases. Therefore, development of these drugs requires that we consider a balance between the promise of a lifesaving treatment and the related risks, known or unknown. Two AAV-based drugs (Luxturna and Zolgensma) were recently approved by the FDA, and several clinical trials using AAV-based therapies are in progress $(144,145)$. The information gathered and the knowledge gained from these trials could shape the future direction of the field.

AAVs are evidenced to be less immunogenic than other viral vector platforms but can still mount a substantially high immune response in a dose-dependent manner. Innate immunity mounts rapidly, is nonspecific, and does not result in immunological memory. The resultant responses of the innate immune system are usually brief and mainly arise as soon as the vectors are administered. Although the activation of innate immunity in humans by rAAVs is still debatable, several occurrences of serious adverse events (SAEs), some leading to fatalities, in AAV gene therapy trials were suspected to involve the innate immune response. Importantly, these reports have yet to be peer-reviewed, so the implication of innate immunity and complement activation is still speculative. In addition, it is vital to note that the encountered SAEs are dosedependent and are only seen in high-dose trials in which rAAV is administered to reach muscles or the CNS.

Despite the roadblocks, AAVs remain one of the most promising tools for therapeutic gene transfer by far. In addition to adopting a multidisciplinary approach, further studies are undoubtedly required to elucidate the complex mechanisms of the innate immune response that is triggered by AAV. Additional preclinical studies are also needed to better predict safer doses and biomarkers of toxicity, as well as the influence of parameters like empty capsid levels, impurities resulting from manufacturing methods, 
and different purification strategies. The greater our understanding of these details, the more interventions and manipulation strategies to improve AAV gene therapy will come to light.

\section{Acknowledgments}

We thank the members of the Gao laboratory for the insightful conversations that made this Review possible. SR is supported by grants from the NIH (R01AI141181, R01AI32296, and R33AI136007). KAF is supported by grants from the NIH (AI067497 and AI128358-04). GG is supported by grants from the University of
Massachusetts Medical School (an internal grant) and from the NIH (R01NS076991-01, 1P01AI100263-01, 4P01HL131471-02, UG3 HL147367-01, R01HL097088, and 1U19AI149646).

Address correspondence to: Guangping Gao or Phillip W.L. Tai, Horae Gene Therapy Center, University of Massachusetts Medical School, 386 Plantation Street, Worcester, Massachusetts 01605, USA. Phone: 508.856.3394; Email: guangping.gao@umassmed. edu (GG). Phone: 774.455.4559; Email: Phillip.Tai2@umassmed. edu (PWLT).
1. Gao G, et al. Clades of adeno-associated viruses are widely disseminated in human tissues. J Virol. 2004;78(12):6381-6388.

2. Balakrishnan B, Jayandharan GR. Basic biology of adeno-associated virus (AAV) vectors used in gene therapy. Curr Gene Ther. 2014;14(2):86-100.

3. Wang D, et al. Adeno-associated virus vector as a platform for gene therapy delivery. Nat Rev Drug Discov. 2019;18(5):358-378.

4. Ogden PJ, et al. Comprehensive AAV capsid fitness landscape reveals a viral gene and enables machine-guided design. Science. 2019;366(6469):11309-1143.

5. Li C, Samulski RJ. Engineering adeno-associated virus vectors for gene therapy. Nat Rev Genet. 2020;21(4):255-272.

6. Mingozzi F, High KA. Therapeutic in vivo gene transfer for genetic disease using AAV: progress and challenges. Nat Rev Genet. 2011;12(5):341-355.

7. Li C, et al. Neutralizing antibodies against adeno-associated virus examined prospectively in pediatric patients with hemophilia. Gene Ther. 2012;19(3):288-294.

8. Calcedo R, et al. Worldwide epidemiology of neutralizing antibodies to adeno-associated viruses. J Infect Dis. 2009;199(3):381-390.

9. Louis Jeune V, et al. Pre-existing anti-adenoassociated virus antibodies as a challenge in AAV gene therapy. Hum Gene Ther Methods. 2013;24(2):59-67.

10. Petry $\mathrm{H}$, et al. Effect of viral dose on neutralizing antibody response and transgene expression after AAV1 vector re-administration in mice. Gene Ther. 2008;15(1):54-60.

11. Manno CS, et al. Successful transduction of liver in hemophilia by AAV-Factor IX and limitations imposed by the host immune response. Nat Med . 2006;12(3):342-347.

12. Flotte TR. Revisiting the "new" inflammatory toxicities of adeno-associated virus vectors. Hum Gene Ther. 2020;31(7-8):398-399.

13. Hordeaux J, et al. Toxicology study of intra-cisterna magna adeno-associated virus 9 expressing human alpha-L-iduronidase in rhesus macaques. Mol Ther Methods Clin Dev. 2018;10:79-88.

14. Hordeaux J, et al. Toxicology study of intracisterna magna adeno-associated virus 9 expressing iduronate-2-sulfatase in rhesus macaques. Mol Ther Methods Clin Dev. 2018;10:68-78.

15. Hinderer $\mathrm{C}$, et al. Severe toxicity in nonhuman primates and piglets following high-dose intravenous administration of an adeno-associated virus vector expressing human SMN. Hum Gene Ther. 2018;29(3):285-298
16. Perez BA, et al. Management of neuroinflammatory responses to $\mathrm{AAV}$-mediated gene therapies for neurodegenerative diseases. Brain Sci. 2020;10(2):E119.

17. Wilson JM, Flotte TR. Moving forward after two deaths in a gene therapy trial of myotubular myopathy. Hum Gene Ther. 2020;31(13-14):695-696.

18. Srivastava A. AAV vectors: are they safe? Hum Gen Ther. 2020;31(13-14):697-699.

19. Audentes therapeutics provides update on the ASPIRO clinical trial evaluating AT132 in patients with X-linked myotubular myopathy. Press release. Audentes Therapeutics. August 20, 2020. https://www.audentestx.com/press release/audentes-therapeutics-providesupdate-on-the-aspiro-clinical-trial-evaluatingat132-in-patients-with-x-linked-myotubularmyopathy/. Accessed November 2, 2020.

20. Holles N, Conner E. Statement from Audentes $\sim$ June 23, 2020. https://www.joshuafrase.org/ get-involved/recensus-study.php. Accessed November 2, 2020.

21. Mendell JR, et al. Single-dose gene-replacement therapy for spinal muscular atrophy. $N$ Engl $J$ Med. 2017;377(18):1713-1722.

22. Audentes announces positive interim data from first dose cohort of ASPIRO, a phase $1 / 2$ clinical trial of AT132 in patients with $\mathrm{X}$-linked myotubular myopathy. News release. Audentes Therapeutics. January 4, 2018. https://www.prnewswire.com/news-releases/ audentes-announces-positive-interim-datafrom-first-dose-cohort-of-aspiro-a-phase12-clinical-trial-of-at132-in-patients-with- $x$ linked-myotubular-myopathy-300577455.html. Accessed November 2, 2020.

23. Hale C. Solid Bio sees yet another clinical hold for its DMD gene therapy. Fierce Biotech. November 12, 2019. https://www.fiercebiotech.com/ biotech/solid-bio-sees-yet-another-clinical-holdfor-its-dmd-gene-therapy. Accessed November $2,2020$.

24. Pfizer's new phase $1 \mathrm{~b}$ results of gene therapy in ambulatory boys with duchenne muscular dystrophy (DMD) support advancement into pivotal phase 3 study. Press release. Pfizer. May 15, 2020. https://investors.pfizer.com/investornews/press-release-details/2020/PfizersNew-Phase-1b-Results-of-Gene-Therapyin-Ambulatory-Boys-with-Duchenne-MuscularDystrophy-DMD-Support-Advancement-intoPivotal-Phase-3-Study/default.aspx. Accessed November 2, 2020.

25. Paulk N. Gene therapy: it's time to talk about high- dose AAV. Genet Eng Biotechnol News. 2020;40(9). https://www.genengnews.com/commentary/ gene-therapy-its-time-to-talk-about-high-doseaav/. Accessed November 2, 2020.

26. Rumachik NG, et al. Methods matter: standard production platforms for recombinant AAV produce chemically and functionally distinct vectors. Mol Ther Methods Clin Dev. 2020;18:98-118.

27. Zaiss AK, et al. Differential activation of innate immune responses by adenovirus and adeno-associated virus vectors. J Virol. 2002;76(9):4580-4590.

28. Hensley SE, et al. Type I interferon inhibits antibody responses induced by a chimpanzee adenovirus vector. Mol Ther. 2007;15(2):393-403.

29. Goubau D, et al. Cytosolic sensing of viruses. Immunity. 2013;38(5):855-869.

30. Rosin DL, Okusa MD. Dangers within: DAMP responses to damage and cell death in kidney disease. J Am Soc Nephrol. 2011;22(3):416-425.

31. Takeuchi O, Akira S. Pattern recognition receptors and inflammation. Cell. 2010;140(6):805-820.

32. Brubaker SW, et al. Innate immune pattern recognition: a cell biological perspective. Annu Rev Immunol. 2015;33:257-290.

33. Ivashkiv LB, Donlin LT. Regulation of type I interferon responses. Nat Rev Immunol. 2014;14(1):36-49.

34. Rogers GL, et al. Unique roles of TLR9- and MyD88-dependent and -independent pathways in adaptive immune responses to AAV-mediated gene transfer. J Innate Immun. 2015;7(3):302-314.

35. Kawai T, Akira S. The role of pattern-recognition receptors in innate immunity: update on Toll-like receptors. Nat Immunol. 2010;11(5):373-384.

36. Chen N, et al. RNA sensors of the innate immune system and their detection of pathogens. IUBMB Life. 2017;69(5):297-304

37. Huang $X$, Yang Y. Innate immune recognition of viruses and viral vectors. Hum Gene Ther. 2009;20(4):293-301.

38. Kawasaki T, Kawai T. Toll-like receptor signaling pathways. Front Immunol. 2014;5:461.

39. Kawai T, Akira S. Toll-like receptors and their crosstalk with other innate receptors in infection and immunity. Immunity. 2011;34(5):637-650

40. Rogers GL, et al. Innate immune responses to AAV vectors. Front Microbiol. 2011;2:194.

41. Werling D, et al. Variation matters: TLR structure and species-specific pathogen recognition. Trends Immunol. 2009;30(3):124-130.

42. Kawai T, Akira S. Signaling to NF-kappaB by Toll-like receptors. Trends Mol Med. 2007;13(11):460-469. 
43. Fitzgerald KA, et al. IKKepsilon and TBK1 are essential components of the IRF3 signaling pathway. Nat Immunol. 2003;4(5):491-496.

44. Kawai $\mathrm{T}$, et al. Interferon-alpha induction through Toll-like receptors involves a direct interaction of IRF7 with MyD88 and TRAF6. Nat Immunol. 2004;5(10):1061-1068.

45. Somanathan S, et al. AAV vectors avoid inflammatory signals necessary to render transduced hepatocyte targets for destructive T cells. Mol Ther. 2010;18(5):977-982.

46. Rabinowitz J, et al. Adeno-associated virus (AAV) versus immune response. Viruses. 2019;11(2):E102.

47. Zhu J, et al. The TLR9-MyD88 pathway is critical for adaptive immune responses to adenoassociated virus gene therapy vectors in mice. JClin Invest. 2009;119(8):2388-2398.

48. Faust SM, et al. CpG-depleted adeno-associated virus vectors evade immune detection. JClin Invest. 2013;123(7):2994-3001.

49. Ashley SN, et al. TLR9 signaling mediates adaptive immunity following systemic AAV gene therapy. Cell Immunol. 2019;346:103997.

50. Rogers GL, Herzog RW. TLR9 and dendritic cells are required for $\mathrm{CD} 8+\mathrm{T}$ cell responses to the AAV capsid. Blood. 2014;124(21):552.

51. Butterfield JSS, et al. TLR9-activating CpG-B ODN but not TLR7 agonists triggers antibody formation to factor IX in muscle gene transfer. Hum Gene Ther Methods. 2019;30(3):81-92.

52 . McCarty DM. Self-complementary AAV vectors; advances and applications. Mol Ther. 2008;16(10):1648-1656.

53. Martino AT, et al. The genome of self-complementary adeno-associated viral vectors increases Tolllike receptor 9-dependent innate immune responses in the liver. Blood. 2011;117(24):6459-6468.

54. Wu T, et al. Self-complementary AAVs induce more potent transgene product-specific immune responses compared to a single-stranded genome. Mol Ther. 2012;20(3):572-579.

55. Appledorn DM, et al. Adenovirus vector-induced innate inflammatory mediators, MAPK signaling, as well as adaptive immune responses are dependent upon both TLR2 and TLR9 in vivo. JImmunol. 2008;181(3):2134-2144.

56. Barbalat R, et al. Toll-like receptor 2 on inflammatory monocytes induces type I interferon in response to viral but not bacterial ligands. Nat Immunol. 2009;10(11):1200-1207.

57. Quigley M, et al. A critical role for direct TLR2MyD88 signaling in CD8 T-cell clonal expansion and memory formation following vaccinia viral infection. Blood. 2009;113(10):2256-2264.

58. Hösel M, et al. Toll-like receptor 2-mediated innate immune response in human nonparenchymal liver cells toward adeno-associated viral vectors. Hepatology. 2012;55(1):287-297.

59. Sudres M, et al. MyD88 signaling in B cells regulates the production of Th1-dependent antibodies to AAV. Mol Ther. 2012;20(8):1571-1581.

60. Chan YK, et al. Reducing AAV-mediated immune responses and pathology in a subretinal pig model by engineering the vector genome. Mol Ther. 2019;27(suppl 1):298.

61. Letizia A, et al. Engineering AAV vectors to reduce $\mathrm{CD} 8+\mathrm{T}$ cell responses following intramuscular injection in mice. Mol Ther. 2019;27(suppl 1):301.

62. Xia P, et al. DNA sensor cGAS-mediated immune recognition. Protein Cell. 2016;7(11):777-791.

63. Sun L, et al. Cyclic GMP-AMP synthase is a cytosolic DNA sensor that activates the type I interferon pathway. Science. 2013;339(6121):786-791.

64. Jønsson KL, et al. IFI16 is required for DNA sensing in human macrophages by promoting production and function of cGAMP. Nat Commun 2017;8:14391.

65. Hornung V, et al. AIM2 recognizes cytosolic dsDNA and forms a caspase-1-activating inflammasome with ASC. Nature. 2009;458(7237):514-518.

66. Mankan AK, et al. Cytosolic RNA:DNA hybrids activate the CGAS-STING axis. EMBOJ. 2014;33(24):2937-2946.

67. Gao P, et al. Cyclic $\left[\mathrm{G}\left(2^{\prime}, 5^{\prime}\right) \mathrm{pA}\left(3^{\prime}, 5^{\prime}\right) \mathrm{p}\right]$ is the metazoan second messenger produced by DNA-activated cyclic GMP-AMP synthase. Cell. 2013;153(5):1094-1107.

68. Andreeva L, et al. cGAS senses long and HMGB/TFAM-bound U-turn DNA by forming protein-DNA ladders. Nature. 2017;549(7672):394-398.

69. Luecke $S$, et al. cGAS is activated by DNA in a length-dependent manner. EMBO Rep. 2017;18(10):1707-1715.

70. Zhang C, et al. Structural basis of STING binding with and phosphorylation by TBK1. Nature. 2019;567(7748):394-398.

71. Li XD, et al. Pivotal roles of cGAS-cGAMP signaling in antiviral defense and immune adjuvant effects. Science. 2013;341(6152):1390-1394.

72. Ishikawa $\mathrm{H}$, et al. STING regulates intracellular DNA-mediated, type I interferon-dependent innate immunity. Nature. 2009;461(7265):788-792.

73. Lum KK, et al. Charge-mediated pyrin oligomerization nucleates antiviral IFI16 sensing of herpesvirus DNA. mBio. 2019;10(4):e01428-19.

74. Jin T, et al. Structures of the HIN domain:DNA complexes reveal ligand binding and activation mechanisms of the AIM2 inflammasome and IFI16 receptor. Immunity. 2012;36(4):561-571.

75. Almine JF, et al. IFI16 and cGAS cooperate in the activation of STING during DNA sensing in human keratinocytes. Nat Commun. 2017;8:14392.

76. Orzalli MH, et al. Nuclear interferon-inducible protein 16 promotes silencing of herpesviral and transfected DNA. Proc Natl Acad Sci U S A. 2013;110(47):E4492-E4501.

77. Chandler LC, et al. Enhancement of adenoassociated virus-mediated gene therapy using hydroxychloroquine in murine and human tissues. Mol Ther Methods Clin Dev. 2019;14:77-89.

78. Ding $\mathrm{W}$, et al. Intracellular trafficking of adeno-associated viral vectors. Gene Ther. 2005;12(11):873-880.

79. Huang LS, et al. mtDNA activates cGAS signaling and suppresses the YAP-mediated endothelial cell proliferation program to promote inflammatory injury. Immunity. 2020;52(3):475-486.e5.

80. Bae JH, et al. Circulating cell-free mtDNA contributes to AIM2 inflammasome-mediated chronic inflammation in patients with type 2 diabetes. Cells. 2019;8(4):E328.

81. Stratmann SA, et al. The innate immune sensor
IFI16 recognizes foreign DNA in the nucleus by scanning along the duplex. Elife. 2015;4:e11721.

82. Xiao TS. The nucleic acid-sensing inflammasomes. Immunol Rev. 2015;265(1):103-111.

83. Dell'Oste V, et al. Innate nuclear sensor IFI16 translocates into the cytoplasm during the early stage of in vitro human cytomegalovirus infection and is entrapped in the egressing virions during the late stage. J Virol. 2014;88(12):6970-6982.

84. Orzalli MH, et al. cGAS-mediated stabilization of IFI16 promotes innate signaling during herpes simplex virus infection. Proc Natl Acad Sci U S A. 2015;112(14):E1773-E1781.

85. Hu B, et al. The DNA-sensing AIM2 inflammasome controls radiation-induced cell death and tissue injury. Science. 2016;354(6313):765-768.

86. Kaminski JJ, et al. Synthetic oligodeoxynucleotides containing suppressive TTAGGG motifs inhibit AIM2 inflammasome activation. J Immunol.2013;191(7):3876-3883.

87. Steinhagen F, et al. Suppressive oligodeoxynucleotides containing TTAGGG motifs inhibit cGAS activation in human monocytes. Eur J Immunol. 2018;48(4):605-611.

88. Schlee M. Master sensors of pathogenic RNA - RIG-I like receptors. Immunobiology. 2013;218(11):1322-1335.

89. Kell AM, Gale M. RIG-I in RNA virus recognition. Virology. 2015;479-480:110-121.

90. Haberman RP, et al. Novel transcriptional regulatory signals in the adeno-associated virus terminal repeat A/D junction element. J Virol. 2000;74(18):8732-8739.

91. Qiu J, et al. Characterization of the transcription profile of adeno-associated virus type 5 reveals a number of unique features compared to previously characterized adeno-associated viruses. JVirol. 2002;76(24):12435-12447.

92. Shao W, et al. Double-stranded RNA innate immune response activation from long-term adeno-associated virus vector transduction. JCI Insight. 2018;3(12):e120474.

93. Reichel FF, et al. AAV8 can induce innate and adaptive immune response in the primate eye. Mol Ther. 2017;25(12):2648-2660.

94. Murai K, et al. Induction of selenoprotein $P$ mRNA during hepatitis $C$ virus infection inhibit RIG-I-mediated antiviral immunity. Cell Host Microbe. 2019;25(4):588-601.e7.

95. Eckard SC, et al. The SKIV2L RNA exosome limits activation of the RIG-I-like receptors. Nat Immunol. 2014;15(9):839-845.

96. Fan J, et al. A human long non-coding RNA LncATV promotes virus replication through restricting RIG-I-mediated innate immunity. Front Immunol. 2019;10:1711.

97. Hoesel M, et al. Innate immune response towards adeno-associated viral (AAV) vectors in human liver non-parenchymal cells. Mol Ther. 2010;18(suppl 1):S120.

98. Calcedo R, et al. Adeno-associated virus antibody profiles in newborns, children, and adolescents. Clin Vaccine Immunol. 2011;18(9):1586-1588.

99. Mingozzi F, et al. Prevalence and pharmacological modulation of humoral immunity to AAV vectors in gene transfer to synovial tissue. Gene Ther. 2013;20(4):417-424.

100.Jiang $\mathrm{H}$, et al. Effects of transient immunosup- 
pression on adenoassociated, virus-mediated, liver-directed gene transfer in rhesus macaques and implications for human gene therapy. Blood. 2006;108(10):3321-3328.

101.Samaranch L, et al. Adeno-associated virus serotype 9 transduction in the central nervous system of nonhuman primates. Hum Gene Ther. 2012;23(4):382-389.

102. Gray SJ, et al. Global CNS gene delivery and evasion of anti-AAV-neutralizing antibodies by intrathecal AAV administration in non-human primates. Gene Ther. 2013;20(4):450-459.

103. Wang D, et al. A rationally engineered capsid variant of AAV9 for systemic CNS-directed and peripheral tissue-detargeted gene delivery in neonates. Mol Ther Methods Clin Dev. 2018;9:234-246.

104. Kotterman MA, et al. Antibody neutralization poses a barrier to intravitreal adeno-associated viral vector gene delivery to non-human primates. Gene Ther. 2015;22(2):116-126.

105. Merle NS, et al. Complement system part Imolecular mechanisms of activation and regulation. Front Immunol. 2015;6:262.

106.Sarma JV, Ward PA. The complement system. Cell Tissue Res. 2011;343(1):227-235.

107. Carroll MC, Isenman DE. Regulation of humoral immunity by complement. Immunity. 2012;37(2):199-207.

108. Clarke EV, Tenner AJ. Complement modulation of $\mathrm{T}$ cell immune responses during homeostasis and disease. J Leukoc Biol. 2014;96(5):745-756.

109.Zaiss AK, et al. Complement is an essential component of the immune response to adeno-associated virus vectors. J Virol. 2008;82(6):2727-2740.

110.Schneider MC, et al. Functional significance of factor $\mathrm{H}$ binding to Neisseria meningitidis. JImmunol. 2006;176(12):7566-7575.

111. Carroll MC. The complement system in regulation of adaptive immunity. Nat Immunol. 2004;5(10):981-986.

112. Carroll MC. The complement system in B cell regulation. Mol Immunol. 2004;41(2-3):141-146.

113. Pfizer presents initial clinical data on phase 1 gene therapy study for duchenne muscular dystrophy (DMD). Press release. Pfizer. June 28, 2019. https://www.pfizer.com/news/pressrelease/press-release-detail/pfizer_presents_ initial_clinical_data_on_phase_1b_gene_therapy_ study_for_duchenne_muscular_dystrophy_dmd. Accessed November 2, 2020.

114. Solid Biosciences provides SGT-001 program update. Press release. Solid Biosciences. November 12, 2019. https://www.solidbio.com/about/ media/press-releases/solid-biosciencesprovides-sgt-001-program-update. Accessed November 2, 2020.

115. Dalkara D, et al. In vivo-directed evolution of a new adeno-associated virus for therapeutic outer retinal gene delivery from the vitreous. Sci Transl Med. 2013;5(189):189ra76.

116. Tse LV, et al. Structure-guided evolution of antigenically distinct adeno-associated virus variants for immune evasion. Proc Natl Acad Sci U S A. 2017;114(24):E4812-E4821.

117. Wobus CE, et al. Monoclonal antibodies against the adeno-associated virus type $2(\mathrm{AAV}-2)$ capsid: epitope mapping and identification of capsid domains involved in AAV-2-cell interaction and neutralization of AAV-2 infection. JVirol. 2000;74(19):9281-9293.

118. Li C, et al. Single amino acid modification of adeno-associated virus capsid changes transduction and humoral immune profiles. JVirol. 2012;86(15):7752-7759.

119. Maheshri N, et al. Directed evolution of adenoassociated virus yields enhanced gene delivery vectors. Nat Biotechnol. 2006;24(2):198-204.

120. Grimm D, et al. In vitro and in vivo gene therapy vector evolution via multispecies interbreeding and retargeting of adeno-associated viruses. JVirol. 2008;82(12):5887-5911.

121. Paulk NK, et al. Bioengineered AAV capsids with combined high human liver transduction in vivo and unique humoral seroreactivity. Mol Ther. 2018;26(1):289-303.

122. Monteilhet $\mathrm{V}$, et al. $\mathrm{A} 10$ patient case report on the impact of plasmapheresis upon neutralizing factors against adeno-associated virus (AAV) types 1, 2, 6, and 8. Mol Ther. 2011;19(11):2084-2091.

123. Chicoine LG, et al. Plasmapheresis eliminates the negative impact of AAV antibodies on microdystrophin gene expression following vascular delivery. Mol Ther. 2014;22(2):338-347.

124. Bertin B, et al. Capsid-specific removal of circulating antibodies to adeno-associated virus vectors. Sci Rep. 2020;10(1):864.

125. Vandamme $C$, et al. Unraveling the complex story of immune responses to AAV vectors trial after trial. Hum Gene Ther. 2017;28(11):1061-1074.

126. Meliani A, et al. Enhanced liver gene transfer and evasion of preexisting humoral immunity with exosome-enveloped AAV vectors. Blood Adv. 2017;1(23):2019-2031.

127. Elmore ZC, et al. Rescuing AAV gene transfer from neutralizing antibodies with an IgG-degrading enzyme. JCI Insight. 2020;5(19):e139881.

128. Leborgne $\mathrm{C}$, et al. IgG-cleaving endopeptidase enables in vivo gene therapy in the presence of anti-AAV neutralizing antibodies. Nat Med. 2020;26(7):1096-1101.

129. Mingozzi F, et al. Overcoming preexisting humoral immunity to AAV using capsid decoys. Sci Transl Med. 2013;5(194):194ra92.

130. Apellis Pharmaceuticals will commence APL-9 program to control the complement system in host responses to AAV vector administration for gene therapies. News release. Apellis Pharmaceuticals. July 18, 2019. https://www.globenewswire.com/news-release/2019/07/18/1884481/0/ en/Apellis-Pharmaceuticals-Will-CommenceAPL-9-Program-to-Control-the-ComplementSystem-in-Host-Responses-to-AAV-VectorAdministration-for-Gene-Therapies.html. Accessed November 2, 2020.

131. Rogers GL, et al. Plasmacytoid and conventional dendritic cells cooperate in crosspriming AAV capsid-specific $\mathrm{CD} 8^{+} \mathrm{T}$ cells. Blood. 2017;129(24):3184-3195.

132. Martino AT, et al. Engineered AAV vector minimizes in vivo targeting of transduced hepatocytes by capsid-specific CD8+ T cells. Blood . 2013;121(12):2224-2233.

133. Li C, et al. Adeno-associated virus capsid antigen presentation is dependent on endosomal escape. J Clin Invest. 2013;123(3):1390-1401.
134.Pei X, et al. Efficient capsid antigen presentation from adeno-associated virus empty virions in vivo. Front Immunol. 2018;9:844.

135. McIntosh JH, et al. Successful attenuation of humoral immunity to viral capsid and transgenic protein following AAV-mediated gene transfer with a non-depleting $\mathrm{CD} 4$ antibody and cyclosporine. Gene Ther. 2012;19(1):78-85.

136. Roche PA, Furuta K. The ins and outs of MHC class II-mediated antigen processing and presentation. Nat Rev Immunol. 2015;15(4):203-216.

137. Nathwani AC, et al. Adenovirus-associated virus vector-mediated gene transfer in hemophilia B. N EnglJMed. 2011;365(25):2357-2365.

138. Nathwani AC, et al. Long-term safety and efficacy of factor IX gene therapy in hemophilia B. NEnglJMed.2014;371(21):1994-2004.

139. He Y, et al. Kinetics of adeno-associated virus serotype 2 (AAV2) and AAV8 capsid antigen presentation in vivo are identical. Hum Gene Ther. 2013;24(5):545-553.

140.Wang L, et al. Systemic protein delivery by muscle-gene transfer is limited by a local immune response. Blood. 2005;105(11):4226-4234.

141. Boisgerault F, et al. Prolonged gene expression in muscle is achieved without active immune tolerance using microrRNA 142.3p-regulated rAAV gene transfer. Hum Gene Ther. 2013;24(4):393-405.

142.Xiao $\mathrm{Y}$, et al. Circumventing cellular immunity by miR142-mediated regulation sufficiently supports rAAV-delivered OVA expression without activating humoral immunity. JCI Insight. 2019;5(13):e99052.

143. Majowicz A, et al. Mir-142-3p target sequences reduce transgene-directed immunogenicity following intramuscular adeno-associated virus 1 vector-mediated gene delivery. J Gene Med. 2013;15(6-7):219-232.

144.Pierce EA, Bennett J. The status of RPE65 gene therapy trials: safety and efficacy. Cold Spring Harb Perspect Med. 2015;5(9):a017285.

145. Hoy SM. Onasemnogene abeparvovec: first global approval. Drugs. 2019;79(11):1255-1262.

146.Ma Z, et al. Innate sensing of DNA virus genomes. Annu Rev Virol. 2018;5(1):341-362.

147. Dell'Oste V, et al. The interferon-inducible DNA-sensor protein IFI16: a key player in the antiviral response. New Microbiol. 2015;38(1):5-20.

148. Nathwani AC, et al. Self-complementary adenoassociated virus vectors containing a novel liver-specific human factor IX expression cassette enable highly efficient transduction of murine and nonhuman primate liver. Blood. 2006;107(7):2653-2661.

149. Wright JF. Codon modification and PAMPs in clinical AAV vectors: the tortoise or the hare? Mol Ther. 2020;28(3):701-703.

150. de Moreuil C, et al. Hydroxychloroquine may be beneficial in preeclampsia and recurrent miscarriage. Br JClin Pharmacol. 2020;86(1):39-49.

151. An J, et al. Cutting edge: antimalarial drugs inhibit IFN- $\beta$ production through blockade of cyclic GMP-AMP synthase-DNA interaction. J Immunol. 2015;194(9):4089-4093.

152. Kuznik A, et al. Mechanism of endosomal TLR inhibition by antimalarial drugs and imidazoquinolines. JImmunol. 2011;186(8):4794-4804.

153. Mukherjee S, Mukherjee U. A comprehen- 
sive review of immunosuppression used for liver transplantation.J Transplant. 2009;2009:701464.

154.Zaiss AK, Muruve DA. Immunity to adenoassociated virus vectors in animals and humans: a continued challenge. Gene Ther. 2008;15(11):808-816.

155. Corti M, et al. Evaluation of readministration of a recombinant adeno-associated virus vector expressing acid alpha-glucosidase in Pompe disease: preclinical to clinical planning. Hum Gene Ther Clin Dev. 2015;26(3):185-193.

156. Meliani A, et al. Antigen-selective modulation of AAV immunogenicity with tolerogenic rapamycin nanoparticles enables successful vector readministration. Nat Commun. 2018;9(1):4098.

157. Maldonado RA, et al. Polymeric synthetic nanoparticles for the induction of antigenspecific immunological tolerance. Proc Natl Acad Sci U S A. 2015;112(2):E156-E165

158. Kishimoto TK, et al. Improving the efficacy and safety of biologic drugs with tolerogenic nanoparticles. Nat Nanotechnol. 2016;11(10):890-899.

159. Mimuro J, et al. Minimizing the inhibitory effect of neutralizing antibody for efficient gene expression in the liver with adeno-associated virus 8 vectors. Mol Ther. 2013;21(2):318-323.

160. Boutin S, et al. Prevalence of serum IgG and neutralizing factors against adeno-associated virus (AAV) types 1, 2, 5, 6, 8, and 9 in the healthy population: implications for gene therapy using AAV vectors. Hum Gene Ther. 2010;21(6):704-712.

161. Legendre CM, et al. Terminal complement inhibitor eculizumab in atypical hemolytic-uremic syndrome. N Engl JMed. 2013;368(23):2169-2181.

162.Trouw LA, et al. The complement system as a potential therapeutic target in rheumatic disease. Nat Rev Rheumatol. 2017;13(9):538-547.

163. Monahan PE, et al. Proteasome inhibitors enhance gene delivery by AAV virus vectors expressing large genomes in hemophilia mouse and dog models: a strategy for broad clinical application. Mol Ther. 2010;18(11):1907-1916.

164. Finn JD, et al. Proteasome inhibitors decrease AAV2 capsid derived peptide epitope presentation on MHC class I following transduction. $\mathrm{Mol}$ Ther. 2010;18(1):135-142.

165.Zhong L, et al. Tyrosine-phosphorylation of AAV2 vectors and its consequences on viral intracellular trafficking and transgene expression. Virology. 2008;381(2):194-202.

166. Gabriel N, et al. Bioengineering of AAV2 capsid at specific serine, threonine, or lysine residues improves its transduction efficiency in vitro and in vivo. Hum Gene Ther Methods. 2013;24(2):80-93.

167. Zhong L, et al. Next generation of adeno-associated virus 2 vectors: point mutations in tyrosines lead to high-efficiency transduction at lower doses. Proc Natl Acad Sci U S A. 2008;105(22):7827-7832.

168. Sun B, et al. Correction of glycogen storage disease type II by an adeno-associated virus vector containing a muscle-specific promoter. Mol Ther. 2005;11(6):889-898.

169.Salva MZ, et al. Design of tissue-specific regulatory cassettes for high-level rAAV-mediated expression in skeletal and cardiac muscle. Mol Ther. 2007;15(2):320-329.

170.Li X, et al. Synthetic muscle promoters: activities exceeding naturally occurring regulatory sequences. Nat Biotechnol.1999;17(3):241-245.

171. Gonin P, et al. Femoral intra-arterial injection: a tool to deliver and assess recombinant AAV constructs in rodents whole hind limb. J Gene Med.
2005;7(6):782-791.

172. Dhungel B, et al. Synergistic and independent action of endogenous microRNAs 122a and 199a for post-transcriptional liver detargeting of gene vectors. Sci Rep. 2018;8(1):15539.

173. Xie J, et al. MicroRNA-regulated, systemically delivered rAAV9: a step closer to CNS-restricted transgene expression. Mol Ther. 2011;19(3):526-535.

174. Qiao C, et al. Liver-specific microRNA-122 target sequences incorporated in $\mathrm{AAV}$ vectors efficiently inhibits transgene expression in the liver. Gene Ther. 2011;18(4):403-410.

175. Brown BD, et al. Endogenous microRNA regulation suppresses transgene expression in hematopoietic lineages and enables stable gene transfer. Nat Med.2006;12(5):585-591.

176. Brown BD, et al. Endogenous microRNA can be broadly exploited to regulate transgene expression according to tissue, lineage and differentiation state. Nat Biotechnol. 2007;25(12):1457-1467.

177. Hengel H, et al. Cytomegaloviral control of MHC class I function in the mouse. Immunol Rev. 1999;168:167-176.

178. Ahn K, et al. Molecular mechanism and species specificity of TAP inhibition by herpes simplex virus ICP47. EMBO J. 1996;15(13):3247-3255.

179. Tomazin R, et al. Herpes simplex virus type 2 ICP47 inhibits human TAP but not mouse TAP. J Virol. 1998;72(3):2560-2563.

180. Lehner PJ, et al. The human cytomegalovirus US6 glycoprotein inhibits transporter associated with antigen processing-dependent peptide translocation. Proc Natl Acad Sci U S A. 1997;94(13):6904-6909.

181. Shao $\mathrm{W}$, et al. Inhibition of antigen presentation during AAV gene therapy using virus peptides. Hum Mol Genet. 2018;27(4):601-613. 